\title{
Multiqubit UPB: The method of formally orthogonal matrices
}

\author{
Lin Chen ${ }^{1,2}$, 田 and Dragomir Ž Đoković 3 , \\ ${ }^{1}$ School of Mathematics and Systems Science, Beihang University, Beijing 100191, China \\ ${ }^{2}$ International Research Institute for Multidisciplinary Science, Beihang University, Beijing 100191, China \\ ${ }^{3}$ Department of Pure Mathematics and Institute for Quantum Computing, \\ University of Waterloo, Waterloo, Ontario, N2L 3G1, Canada
}

(Dated: August 31, 2018)

\begin{abstract}
We use formal matrices whose entries we view as vector variables taking unit vectors values in one-qubit Hilbert spaces of a multiqubit quantum system. We construct many unextendible product bases (UPBs) of new sizes in such systems and provide a new construction of UPBs of $n$ qubits of cardinality $n+1$ when $n \equiv 3(\bmod 4)$. We also give a new method of constructing multiqubit entangled states with all partial transposes positive.
\end{abstract}

PACS numbers: 03.65.Ud, 03.67.Mn

* linchen@buaa.edu.cn (corresponding author)

$\dagger$ djokovic@uwaterloo.ca 


\section{CONTENTS}

\section{Introduction}

II. Basic concepts and definitions

III. Evaluations and extensions of orthogonal matrices

IV. Old and new facts about multiqubit UPBs

VI. Construction of multiqubit PPT entangled states

VII. A partial order in $\mathcal{M}(m, n)$

Acknowledgements

References

VIII. Appendix A

IX. Appendix B

\section{INTRODUCTION}

We introduced in [9] a formal matrix approach in order to study the orthogonal product bases (OPB) in multiqubit systems. We applied this method to obtain a coarse classification of OPBs of four qubits. We warn the reader that this is not the classification under local unitary transformations and qubit permutations.

In the present paper we extend this formal approach in order to study the unextendible product bases (UPB) in multiqubit systems. We adapted many definitions from [9] and introduced some new ones. The entries of our formal matrices, $X$, are vector variables which take unit vector values (up to the phase factor) in one of the single qubit Hilbert spaces. The column $j$ of the matrix corresponds to the $j$ th qubit.

The powerful tool of so called orthogonality graphs is naturally embedded into our formal matrix framework. Each vector variable, say $x$, has a companion, its perpendicular, which we denote by $x^{\prime}$. If $x$ takes a value $|a\rangle$ then $x^{\prime}$ takes as its value the unique unit vector $|a\rangle^{\perp}$ orthogonal to $|a\rangle$, in the Hilbert space of the same qubit. In this way the rows of the formal matrix $X$ give rise to product vectors. The rows of $X$ are the vertices of the orthogonality graphs. We obtain the orthogonality graph of the $j$ th qubit by joining the vertices $i$ and $k$ if and only if the matrix entries in the positions $(i, j)$ and $(k, j)$ are the perpendiculars of each other. The rows $i$ and $k$ are orthogonal if such $j$ exists. The matrix is orthogonal if any two of its rows are orthogonal. An orthogonal matrix $X$ is an unextendible orthogonal matrix (UOM) if there is no row orthogonal to all of the rows of $X$. (See the next section for precise definitions.) Each UOM $X$ gives an infinite family of UPBs, which we denote by $\mathcal{F}_{X}^{\#}$, having the same orthogonality graphs as $X$.

The formal method makes it possible to simplify the proofs of many known facts, to generalize some of the known results, and is conducive to making new constructions and conjectures. For instance, by using Lemma 15 in Sec. VI we construct a new class of PPTES (entangled states, all of whose partial transposes are positive semidefinite) which we call secondary PPTES.

One of the main open problems in the study of the UPBs in $n$-qubit quantum systems is to determine the set $\Theta_{n}$ of possible sizes of UPBs for fixed $n$. In the language of formal matrices, this set is the set of integers $m$ for which there exist a UOM of size $m \times n$. Many results about the sets $\Theta_{n}$ are known. In particular, the smallest integer $\theta_{n}$ of $\Theta_{n}$ is known [16, 17]. On the other hand, many problems were left open in these papers. One of them was whether $2^{n}-5 \in \Theta_{n}$, to which a negative answer was obtained recently 9 ]. For $n \leq 7$, the question whether $m \in \Theta_{n}$ was left open also when $(m, n)$ is one of the following pairs: $(11,5),(10,6),(11,6),(13,6),(10,7),(11,7),(13,7),(14,7)$, $(15,7),(19,7)$. We have constructed many UOMs of new sizes $m \times n$, in particular for all pairs listed above except $(10,7)$ and $(11,7)$ which remain open. As a consequence, the sets $\Theta_{n}$ are now known exactly for $n \leq 6$ and for $n=8$. Previously they were known only for $n \leq 4$.

The UPBs provide nice examples of PPTES. It is well known that the projector $\rho$ onto the subspace of $\mathcal{H}$ orthogonal to a UPB, say $\mathcal{U}$, is a (non-normalized) PPTES. The range of $\rho$ contains no product vectors. We modify this construction as follows. Let us drop from $\mathcal{U}$ one of the product vectors and denote by $\sigma$ the projector onto the 
subspace orthogonal to the remaining product vectors of $\mathcal{U}$. We show that the range of $\sigma$ contains only finitely many product vectors (up to scalar mutiples). In many cases (but not always) $\sigma$ is a (non-normalized) PPTES. Its range contains at least one product vector, and so these new PPTES are essentially different from the PPTES $\rho$ mentioned above. Our Proposition [19] in Sec. [X] shows that this construction of PPTES is also applicable to UPBs in arbitrary finite-dimensional quantum systems.

The rest of the paper is organized as follows. In Sec. [we generalize our formal matrix approach from [9] in order to be able to apply it to the more general case of arbitrary UPBs (in multiqubit systems). We describe the basic concepts and give the definitions used in this paper, such as orthogonal product bases, vector variables, orthogonal matrices, and equivalence of matrices. In Sec. III we introduce the concepts of the evaluations and extensions of orthogonal matrices, define the UOMs and describe a method of constructing larger orthogonal matrices or UOMs from the smaller ones, see Proposition 7 In Sec. IV Theorem 11, we review some known facts on the existence of UPBs and rephrase them in terms of UOMs. The UOMs of new sizes that we have constructed are listed in Table IV] It is important to have a good test for checking whether two UOMs of the same size are equivalent. The test that we used is based on Lemma 13, In Sec. $\nabla$ we describe a new construction of orthogonal matrices which can be used to produce new UOMs. For instance, for any $n \equiv 3(\bmod 4)$ we can construct new UOMs of size $(n+1) \times n$. In particular, for $n=7$ our construction gives four non-equivalent UOMs. (There are only 7 equivalence classes of UOMs of that size.) In Sec. VI we describe a new type of PPTES associated to UPBs to which we refer as the secondary PPTES. In the case of four qubits, we list in Table III all pairs $(\operatorname{rank} \rho, s)$ where $\rho$ is a secondary PPTES and $s$ is the number of product vectors in the range of $\rho$. In Sec. VII we define a partial order in $\mathcal{M}(m, n)$ and $\mathcal{O}(m, n)$, and use it to deduce a partial order on the set of equivalence classes of orthogonal matrices as well as the equivalence classes of UOMs.

\section{BASIC CONCEPTS AND DEFINITIONS}

Let $\mathcal{H}=\mathcal{H}_{1} \otimes \cdots \otimes \mathcal{H}_{n}$ be the Hilbert space representing a quantum system $A_{1}, \cdots, A_{n}$ consisting of $n$ qubits. Each $\mathcal{H}_{j}$ is a 2-dimensional Hilbert space. We fix an orthonormal basis $|0\rangle_{j},|1\rangle_{j}$ of $\mathcal{H}_{j}$. Usually, the subscript $j$ will be suppressed. We say that a vector $|v\rangle \in \mathcal{H}$ is a unit vector if $\|v\|=1$. For any nonzero vector $\left|v_{j}\right\rangle \in \mathcal{H}_{j}$ we denote by $\left[v_{j}\right]$ the 1-dimensional subspace of $\mathcal{H}_{j}$ spanned by this vector. As a rule, we shall not distinguish two unit vectors which differ only in the phase. By using this convention, we can say that for any unit vector $\left|v_{j}\right\rangle \in \mathcal{H}_{j}$ there exists a unique unit vector $\left|v_{j}\right\rangle^{\perp} \in \mathcal{H}_{j}$ which is perpendicular to $\left|v_{j}\right\rangle$.

A product vector is a nonzero vector $|x\rangle=\left|x_{1}\right\rangle \otimes \cdots \otimes\left|x_{n}\right\rangle$, which will be written also as $|x\rangle=\left|x_{1}, \ldots, x_{n}\right\rangle$. If $\|x\|=1$ we shall assume (as we may) that each $\left\|x_{j}\right\|=1$. Two product vectors $|x\rangle=\left|x_{1}, \ldots, x_{n}\right\rangle$ and $|y\rangle=\left|y_{1}, \ldots, y_{n}\right\rangle$ are orthogonal if and only if $\left|y_{j}\right\rangle=\left|x_{j}\right\rangle^{\perp}$ for at least one index $j$. We use the abbreviation OPS to denote any set of pairwise orthogonal unit product vectors in $\mathcal{H}$. The cardinality of an OPS cannot exceed $2^{n}$, the dimension of $\mathcal{H}$. We say that an OPS is an OPB, orthogonal product basis, if its cardinality is $2^{n}$. As an example, the $2^{n}$ product vectors $\left|x_{s}\right\rangle=\left|s_{1}, \ldots, s_{n}\right\rangle$, where $s:=\left(s_{1}, \ldots, s_{n}\right)$ runs through all binary $\{0,1\}$-sequences of length $n$, is an OPB. We refer to this OPB as the standard OPB. However, there are many other OPBs and describing or classifing them for any $n$ is a very hard problem. Let us also mention that a set of unit product vectors is called an unextendible product basis (UPB) if these vectors are orthogonal to each other and there is no product vector orthogonal to all of them [2, 10]. Originally it was required that UPB does not span the whole Hilbert space $\mathcal{H}$, but for us it is convenient to drop that restriction. We shall say that a UPB is proper if it does not span $\mathcal{H}$.

The above mentioned problem has been considered in [2-4, $, 9,10,[16]$ and in our paper [8] where we studied the case $n=4$. For any $n$, we have reduced this classification problem to a purely combinatorial problem. In order to extend this study further, we need to give some basic definitions.

We start with an infinite countable alphabet $\mathbf{X}$. We shall use letters (with indices if necessary) to denote the elements of this alphabet. The alphabet is equipped with a fixed-point-free involution $x \rightarrow x^{\prime}$. Thus, for any $x \in \mathbf{X}$ we have $x^{\prime} \in \mathbf{X}, x^{\prime} \neq x$ and $\left(x^{\prime}\right)^{\prime}=x$. We shall refer to $x^{\prime}$ as the perpendicular of $x$. We say that a subset of $\mathbf{X}$ is independent if it does not contain any pair of the form $\left\{x, x^{\prime}\right\}$. We shall also say that two vector varables $x$ and $y$ are independent if $x \neq y$ and $x \neq y^{\prime}$. We shall refer to the letters $x \in \mathbf{X}$ as vector variables.

Let $x=\left[\begin{array}{llll}x_{1} & x_{2} & \cdots & x_{n}\end{array}\right]$ and $y=\left[\begin{array}{llll}y_{1} & y_{2} & \cdots & y_{n}\end{array}\right]$ be two row vectors whose entries $x_{j}$ and $y_{j}$ are vector variables. We say that $x$ and $y$ are orthogonal to each other, $x \perp y$, if $y_{j}=x_{j}^{\prime}$ for at least one index $j$.

Next, consider an $m \times n$ matrix $X=\left[x_{i, j}\right]$ with entries $x_{i, j} \in \mathbf{X}$. We say that such a matrix is orthogonal if any two of its rows are orthogonal to each other. (Note that this is different from the traditional definition of orthogonal matrices in linear algebra).

We denote by $\mathcal{M}(m, n)$ the set of all $m \times n$ matrices whose entries belong to $\mathbf{X}$ and satisfy the following additional condition: if a vector variable $x$ occurs in some column of $X$ then neither $x$ nor $x^{\prime}$ occurs in any other column of $X$. We denote by $\mathcal{O}(m, n)$ the subset of $\mathcal{M}(m, n)$ consisting of all orthogonal matrices. We also set $\mathcal{O}(n):=\mathcal{O}\left(2^{n}, n\right)$ 
for the special case $m=2^{n}$. For two matrices $X_{i} \in \mathcal{M}\left(m_{i}, n\right), i=1,2$, we say they are orthogonal to each other, $X_{1} \perp X_{2}$, if each row of $X_{1}$ is orthogonal to each row of $X_{2}$.

If $X=\left[x_{i, j}\right] \in \mathcal{M}(m, n)$ and $x \in \mathbf{X}$ we define the multiplicity, $\mu(x, X)$, of $x$ in $X$ to be the number of pairs $(i, j)$ such that $x_{i, j}=x$. Thus if $x$ does not occur in $X$ then $\mu(x, X)=0$. When $X$ is known from the context we shall simplify this notation by writing just $\mu(x)$. It is easy to see that all maximal independent sets of vector variables, all of which occur in column $j$ of $X$, have the same cardinality. We denote this cardinality by $\nu_{j}(X)$. Finally, we set $\mu(X)=\max _{i, j} \mu\left(x_{i, j}, X\right)$ and $\nu(X)=\sum \nu_{j}(X)$.

We say that a matrix $X \in \mathcal{M}(m, n)$ is balanced if $\mu(x, X)=\mu\left(x^{\prime}, X\right)$ for all vector variables $x$. It is obvious that $X$ is not balanced if $m$ is odd. We have shown in [8] that all UOM in $\mathcal{O}(n)$ are necessarily balanced.

Another important concept is the equivalence of matrices. We say that two matrices $X, Y \in \mathcal{M}(m, n)$ are equivalent if $X$ can be transformed to $Y$ by permuting the rows, permuting the columns, and by renaming the vector variables. The renaming must respect the orthogonality, i.e., we require that if a vector variable $x$ is renamed to $y$ then $x^{\prime}$ has to be renamed to $y^{\prime}$. For $X \in \mathcal{M}(m, n)$ we shall denote by $[X]$ its equivalence class. Note that if $X \in \mathcal{O}(m, n)$ then $[X] \subseteq \mathcal{O}(m, n)$. Since $\mathbf{X}$ is infinite, there are infinitely many matrices in $\mathcal{M}(m, n)$. On the other hand, there are only finitely many equivalence classes in $\mathcal{M}(m, n)$.

Let us give a few very simple examples of orthogonal matrices. The set $\mathcal{O}(1)$ has only one equivalence class, and $\mathcal{O}(2)$ has two classes. Their representatives are

$$
\left[\begin{array}{c}
a \\
a^{\prime}
\end{array}\right], \quad\left[\begin{array}{cc}
a & b \\
a & b^{\prime} \\
a^{\prime} & b \\
a^{\prime} & b^{\prime}
\end{array}\right], \quad\left[\begin{array}{cc}
a & b \\
a & b^{\prime} \\
a^{\prime} & c \\
a^{\prime} & c^{\prime}
\end{array}\right] .
$$

(It is tacitly assumed that $a, b, c \in \mathbf{X}$.)

\section{EVALUATIONS AND EXTENSIONS OF ORTHOGONAL MATRICES}

We say that a matrix $Y \in \mathcal{O}(m, n)$ is an extension of $X \in \mathcal{O}(p, n)$ if $X$ is a submatrix of $Y$. More precisely, we shall say that in this case $Y$ is a $k$-extension of $X$ where $k=m-p$. So the 0 -extension (i.e., the trivial extension) of $X$ is $X$ itself. Note also that $X \in \mathcal{O}(n)$ has no nontrivial extensions. We say that a matrix $X \in \mathcal{O}(m, n)$ is extendible if it has a 1-extension and otherwise we say that it is unextendible. We shall use the abbreviation UOM for "unextendible orthogonal matrix". For example, every $X \in \mathcal{O}(n)$ is a UOM. If $X \in \mathcal{O}(m, n)$ is a UOM and $m<2^{n}$, then we shall say that $X$ is a proper $U O M$.

We say that two extensions $Y_{1}$ and $Y_{2}$ of $X \in \mathcal{O}(m, n)$ are equivalent extensions of $X$ if the matrices $Y_{1}$ and $Y_{2}$ are equivalent. We shall count the number of extensions up to equivalence.

To each orthogonal matrix we can associate a family of OPS. For this purpose we need the concept of evaluation of matrices.

Definition 1 An evaluation of the matrix $X \in \mathcal{M}(m, n)$ is a mapping $\alpha$ which assigns to each vector variable $x$, which occurs in $X$, a unit vector $\alpha(x)$ subject to the following two conditions:

(i) if $x$ occurs in column $j$ of $X$ (recall that such $j$ is unique) then $\alpha(x) \in \mathcal{H}_{j}$;

(ii) if both $x$ and $x^{\prime}$ occur in $X$ (necessarily in the same column) then $\alpha\left(x^{\prime}\right)=\alpha(x)^{\perp}$.

We say that the evaluation $\alpha$ of $X$ is generic if also the following condition is satisfied

(iii) if $x$ and $y$ are independent vector variables in the same column of $X$, then $\alpha(y) \neq \alpha(x)$ and $\alpha(y) \neq \alpha\left(x^{\prime}\right)$.

Note that the generic evaluations $\alpha$ are one-to-one, i.e., if $x \neq y$ then also $\alpha(x) \neq \alpha(y)$. Indeed, let $x$ and $y$ be two different vector variables which occur in $X$. If $x$ and $y$ occur in different columns of $X$ then $\alpha(x) \neq \alpha(y)$ because $\alpha(x)$ and $\alpha(y)$ belong to different Hilbert spaces $\mathcal{H}_{j}$. Now assume that $x$ and $y$ occur in the same column of $X$. If they are independent, then (iii) implies that $\alpha(x) \neq \alpha(y)$. If $x$ and $y$ are dependent, then we have $y=x^{\prime}$ and (ii) implies that $\alpha(y)=\alpha(x)^{\perp} \neq \alpha(x)$.

Given an evaluation $\alpha$ of $X \in \mathcal{M}(m, n)$, we obtain the $m \times n$ matrix $\alpha(X):=\left[\alpha\left(x_{i, j}\right)\right]$ whose entries, $\alpha\left(x_{i, j}\right) \in \mathcal{H}_{j}$, are unit vectors. We shall refer to the matrix $\alpha(X)$ also as evaluation of $X$. Given this evaluation, we can form the product vectors $\alpha\left(x_{i, 1}\right) \otimes \cdots \otimes \alpha\left(x_{i, n}\right), i=1, \ldots, m$. If the matrix $X$ is orthogonal, it is evident that these product vectors form an OPS of cardinality $m$. We refer to it as the OPS of $\alpha(X)$. As the cardinality of an OPS cannot exceed $2^{n}$, we deduce that $\mathcal{O}(m, n)$ is empty for $m>2^{n}$. If $X \in \mathcal{O}(n)$ then the above OPS is in fact an OPB.

To any $X \in \mathcal{O}(m, n)$ we associate a family of OPS which arises from $X$ by applying all evaluations. We denote this family by $\mathcal{F}_{X}$. Further, we denote by $\mathcal{F}_{X}^{\#}$ the subfamily of $\mathcal{F}_{X}$ which arises from $X$ by applying only the generic evaluations. In the case $m=2^{n}$ these two families consist of OPBs. For each $n$, up to equivalence there is exactly 
one matrix $X \in \mathcal{O}(n)$ such that $\mathcal{F}_{X}=\mathcal{F}_{X}^{\#}$. This is the matrix which in each column has just two vector variables, perpendicular to each other, and each with multiplicity $2^{n-1}$.

If $\alpha$ is any evaluation of an extendible orthogonal matrix $X$, then it is obvious that the OPS of $\alpha(X)$ is not a UPB. We consider next the case when $X$ is unextendible and $\alpha$ is generic.

Lemma 2 Let $\alpha$ be a generic evaluation of an $U O M X=\left[x_{i, j}\right] \in \mathcal{O}(m, n)$. Then

(i) the OPS of $\alpha(X)$ is a UPB;

(ii) each member of the family $\mathcal{F}_{X}^{\#}$ is a UPB.

Proof. (i) Assume that the OPS of $\alpha(X)$ is not a UPB. Then there exists a product vector, say $|b\rangle=\left|b_{1}\right\rangle \otimes \cdots \otimes\left|b_{n}\right\rangle$, which is orthogonal to all product vectors $\left|a_{i}\right\rangle:=\alpha\left(x_{i, 1}\right) \otimes \cdots \otimes \alpha\left(x_{i, n}\right), i=1,2, \ldots, m$.

We shall construct a new row $y=\left[\begin{array}{llll}y_{1} & y_{2} & \cdots & y_{n}\end{array}\right]$ of vector variables. For each index $i$ there exists at least one index $j_{i}$ such that $\left|b_{j_{i}}\right\rangle \perp \alpha\left(x_{i, j_{i}}\right)$. We assume that $j_{i}$ is chosen to be the smallest such index. Then we set $y_{j_{i}}:=x_{i, j_{i}}^{\prime}$ for $i=1, \ldots, m$. It may happen that $j_{i}=j_{k}$ for some $k \neq i$. However in that case we have $\alpha\left(x_{i, j_{i}}\right)=\alpha\left(x_{k, j_{i}}\right)$, and so $x_{i, j_{i}}=x_{k, j_{i}}$ because $\alpha$ is generic. Thus the vector variable $y_{j_{i}}$ is well-defined for each index $j_{i}$. It may happen that the set of indices $J:=\left\{j_{i}: i=1, \ldots, m\right\}$ is a proper subset of $\{1,2, \ldots, n\}$. If $j \notin J$ then $y_{j}$ is taken to be a new variable independent from the variables which occur in $X$.

It is immediate from this construction that $y \perp X$. As $X$ is unextendible, we have a contradiction. We conclude that our assumption is false, i.e., the OPS of $\alpha(X)$ must be a UPB.

(ii) follows from (i) and the definition of $\mathcal{F}_{X}^{\#}$.

The following lemma shows that each UPB arises as a generic evaluation of some UOM.

Lemma 3 For each $U P B$, say $\mathcal{U}$, there exists an $U O M X$ such that $\mathcal{U} \in \mathcal{F}_{X}^{\#}$.

Proof. Let $\mathcal{U}$ consist of $m$ product vectors $\left|a_{i}\right\rangle:=\left|a_{i, 1}\right\rangle \otimes \cdots \otimes\left|a_{i, n}\right\rangle, i=1,2, \ldots, m$. We first construct the $m \times n$ matrix $A$ whose entries are the unit vectors $A_{i, j}:=\left|a_{i, j}\right\rangle$. Next, we choose an $m \times n$ matrix of independent vector variables $X=\left[x_{i, j}\right]$ on which we impose only the following relations:

(i) if $\left|a_{i, j}\right\rangle=\left|a_{k, j}\right\rangle$ then $x_{i, j}=x_{k, j}$;

(ii) if $\left|a_{i, j}\right\rangle=\left|a_{k, j}\right\rangle^{\perp}$ then $x_{i, j}=x_{k, j}^{\prime}$.

(We remind the reader that we consider two unit vectors as equal if they differ only in phase.) Then $X \in \mathcal{O}(m, n)$ and $\mathcal{U} \in \mathcal{F}_{X}^{\#}$. Lemma 2 implies that $X$ is unextendible.

In view of the two lemmas above many results about UPBs of multiqubit systems can be expressed in the language of UOMs and vice versa.

For instance, our definition of equivalence in Sec. II (restricted to the UOMs) is compatible with the definition of equivalence of the UPBs in multiqubit systems as defined in [17, p. 4]. More precisely, let $X, Y \in \mathcal{O}(m, n)$ be UOMs and let $\mathcal{U} \in \mathcal{F}_{X}^{\#}$ and $\mathcal{V} \in \mathcal{F}_{Y}^{\#}$. Then $[X]=[Y]$ (i.e., $X$ and $Y$ are equivalent) if and only if the UPBs $\mathcal{U}$ and $\mathcal{V}$ are equivalent.

A general necessary and sufficient condition for a set of orthogonal product states (in any finite-dimensional quantum system) to be unextendible has been obtained in [10, Lemma 1]. In the case of qubit systems, that result has the following simple form.

Lemma 4 A matrix $X \in \mathcal{O}(m, n)$ is extendible if and only if there exist vector variables $y_{1}, \ldots, y_{n}$ such that, for each $j, y_{j}$ occurs in column $j$ of $X$ and each row of $X$ contains at least one of the $y_{j} s$.

For each positive integer $n$ we denote by $\Theta_{n}$ the subset of $\left\{1,2, \ldots, 2^{n}\right\}$ consisting of integers $m$ such that $\mathcal{O}(m, n)$ contains at least one UOM. In view of Lemma 2 and the comments made above, $\Theta_{n}$ is just the set of all sizes of UPBs of $n$ qubits. For instance, we have $\Theta_{1}=\{2\}, \Theta_{2}=\{4\}$ and $\Theta_{3}=\{4,8\}$, and note that $2^{n} \in \Theta_{n}$ for all $n$. In general, it is hard to determine whether $m \in \Theta_{n}$. 
Let us give three examples of UOMs:

$$
X=\left[\begin{array}{ccc}
a & c & e \\
a^{\prime} & d^{\prime} & f \\
b & c^{\prime} & f^{\prime} \\
b^{\prime} & d & e^{\prime}
\end{array}\right], \quad Y=\left[\begin{array}{cccc}
x & y & z & w \\
x^{\prime} & b & d & e \\
a & y^{\prime} & d^{\prime} & f \\
a^{\prime} & c & z^{\prime} & e^{\prime} \\
a & b^{\prime} & d & w^{\prime} \\
x^{\prime} & c^{\prime} & d^{\prime} & f^{\prime}
\end{array}\right], \quad Z=\left[\begin{array}{cccccccc}
a & e & i & m & q & v & \alpha & \zeta \\
b & f & j & m^{\prime} & r & w & \beta & \eta \\
c & g & k & n & s & v^{\prime} & \beta^{\prime} & \theta \\
a^{\prime} & f^{\prime} & l & n^{\prime} & t & x & \gamma & \iota \\
c^{\prime} & h & j^{\prime} & o & t^{\prime} & y & \alpha^{\prime} & \kappa \\
b^{\prime} & h & i^{\prime} & p & s^{\prime} & y^{\prime} & \delta & \iota^{\prime} \\
b^{\prime} & h^{\prime} & l^{\prime} & n^{\prime} & u & z & \epsilon & \zeta^{\prime} \\
c^{\prime} & h^{\prime} & i^{\prime} & p & r^{\prime} & z^{\prime} & \delta & \iota^{\prime} \\
a^{\prime} & f^{\prime} & l^{\prime} & p^{\prime} & t & x & \epsilon^{\prime} & \theta^{\prime} \\
d & g^{\prime} & l & p^{\prime} & q^{\prime} & w^{\prime} & \gamma^{\prime} & \kappa^{\prime} \\
d^{\prime} & e^{\prime} & k^{\prime} & o^{\prime} & u^{\prime} & x^{\prime} & \delta^{\prime} & \eta^{\prime}
\end{array}\right] .
$$

These matrices are obtained from the known three-qubit, four-qubit and eight-qubit UPBs (see [3, 12, 16]). The UOMs $X$ and $Y$ are unique up to equivalence [17], but it is not known whether $Z$ is unique. Further, $X$ is balanced while $Y$ and $Z$ are not.

In the following lemma we collect some basic properties of UOMs.

Lemma 5 Let $X=\left[x_{i, j}\right] \in \mathcal{O}(m, n)$ be a UOM. Then

(i) $x_{i, j}^{\prime}$ occurs in $X$ for all $i, j$;

(ii) if the vector variables $y_{1}, y_{2}, \ldots, y_{s}$ occur in $s$ different columns of $X$, then $X$ has at least $n-s+1$ rows containing no $y_{j}$;

(For $s=0$ we obtain that $m>n$, and for $s=1$ we obtain that $\mu(X) \leq m-n$.)

(iii) for each $i, \sum_{j=1}^{n} \mu\left(x_{i, j}^{\prime}\right) \geq m-1$ and the equality holds if and only if $x_{k, j}=x_{i, j}^{\prime}$ implies that $x_{k, s} \neq x_{i, s}^{\prime}$ whenever $s \neq j$;

(iv) there is an index $r$ such that $x_{r 1}=x_{11}^{\prime}$ and $x_{r s} \neq x_{1 s}^{\prime}$ for $s>1$;

(v) if $Y \in \mathcal{O}(d, k), k<n$, is a submatrix of $X$ then $d \leq m-n+k$ and for $k \geq n / 2$ we have $d<m-n+k$;

(vi) if $p_{j}=\sum \mu(x) \mu\left(x^{\prime}\right)$, where the summation is over all pairs $\left\{x, x^{\prime}\right\}$ in column $j$ of $X$, then $\sum p_{j} \geq m(m-1) / 2$.

Proof. (i) Assume that say $\mu\left(x_{1,1}^{\prime}\right)=0$. Then $\left[x_{1,1}^{\prime} x_{1,2} \cdots x_{1, n}\right] \perp X$, and we have a contradiction.

(ii) Denote by $r$ the number of rows of $X$ containing no $y_{j}$. Suppose that $r \leq n-s$. By permuting the rows and columns of $X$, we may assume that $y_{j}$ occurs in column $j$ for each $j$ and that the first $r$ rows contain no $y_{j}$. Then the row $\left[y_{1}^{\prime} \cdots y_{s}^{\prime} x_{1,1+s}^{\prime} \cdots x_{r, r+s}^{\prime} x_{1, r+s+1} \cdots x_{1, n}\right]$ is orthogonal to each row of $X$. As $X$ is a UOM, we have a contradiction. We conclude that $r>n-s$.

(iii) The inequality follows from the fact that the row $i$ of $X$ is orthogonal to each of the other $m-1$ rows.

(iv) Let us write the first row of $X$ as $v:=\left[\begin{array}{ll}x_{11} & u\end{array}\right]$, where $u=\left[\begin{array}{lll}x_{12} & \cdots & x_{1 n}\end{array}\right]$, and set $w:=\left[\begin{array}{ll}x_{11}^{\prime} & u\end{array}\right]$. If $w$ is a row of $X$, we can take $r$ to be the index of that row. In that case the assertion obviously holds. We may now assume that $w$ is not a row of $X$. Since $X$ has no 1-extensions, there is a row of $X$, say row $r$, which is not orthogonal to $w$. Therefore $x_{r 1} \neq x_{11}$ and $u$ is not orthogonal to [ $x_{r 2} \cdots x_{r n}$ ]. Hence, $r>1$ and the orthogonality of rows 1 and $r$ of $X$ implies that $x_{r 1}=x_{11}^{\prime}$. This completes the proof.

(v) We may assume that $X=\left[\begin{array}{ll}Y & P \\ Q & R\end{array}\right]$. Let $y$ and $p$ be the first rows of $Y$ and $P$, respectively. If $d>m-n+k$ then $m-d<n-k$ and we can choose a row $z$ of length $n-k$ which is orthogonal to $p$ and $R$. Then $[y z] \perp X$, which gives a contradiction. We conclude that $d \leq m-n+k$.

Suppose now that $k \geq n / 2$. Assume that $d=m-n+k$. If a row of $Y$ is orthogonal to a row of $Q$, say the first rows $y$ and $q$ of these matrices, then there exists a row $z$ of length $n-k$ which is orthogonal to the first row $p$ of $P$ and all the rows of $R$ except the first. Then $[q z] \perp X$ and we have a contradiction. We conclude that no row of $Y$ is orthogonal to a row of $Q$. As $X$ is orthogonal, we must have $Q \perp R$. Since $Q$ has $n-k$ rows and $n-k \leq k$, there exists a row $s \perp Q$. Hence, if $r$ is a row of $R$ then $[s r] \perp X$ and we have a contradiction. We conclude that $d<m-n+k$.

(vi) This follows from the fact that for each pair $(i, k), i<k$, the rows $i$ and $k$ of $X$ are orthogonal to each other. See also [17, Appendix A].

We remark that Lemma 5 (i) is equivalent to [16, Lemma 2]. The inequality $\mu(X) \leq m-n$ mentioned in part (ii) of Lemma 5 has been proved in [17, Appendix A, p. 16].

Corollary 6 If $X \in \mathcal{O}(n+1, n)$, $n$ odd, then $X$ is a UOM if and only if $\mu(X)=1$. 
Proof. The condition is necessary by Lemma 5 (iii).

Suppose that $\mu(X)=1$. Assume that there exists a row $y=\left[\begin{array}{llll}y_{1} & y_{2} & \cdots & y_{n}\end{array}\right] \perp X$. Then we must have $\sum \mu\left(y_{j}^{\prime}, X\right) \geq n+1$. As each $\mu\left(y_{j}^{\prime}, X\right) \leq 1$, we have a contradiction. Hence, there is no such $y$ which means that $X$ is a UOM.

Let $A \in \mathcal{M}(r, s)$ and $B \in \mathcal{M}(m, n-s), n>s$, and assume that any vector variables $x$ and $y$ of $A$ and $B$, respectively, are independent. Further, let $B$ be partitioned into blocks $B_{k} \in \mathcal{M}\left(m_{k}, n-s\right), k=1, \ldots, r$,

$$
B=\left[\begin{array}{c}
B_{1} \\
\vdots \\
B_{r}
\end{array}\right]
$$

Given such data, we denote by

$$
A \models\left(B_{1}, B_{2}, \ldots, B_{r}\right)
$$

the matrix $[\tilde{A} B] \in \mathcal{M}(m, n)$, where $\tilde{A}$ is the $m \times s$ matrix obtained from $A$ by replacing, for each $k$, the row $k$ of $A$ by $m_{k}$ copies of that row. Note that if a vector variable $x$ occurs in one of the blocks $B_{k}$ then $x$ or $x^{\prime}$ may occur in another block but necessarily in the same column.

It is straightforward to verify that if $A$ and the $B_{k}$ are orthogonal matrices, then the matrix $A \models\left(B_{1}, B_{2}, \ldots, B_{r}\right)$ is also orthogonal.

Let us give an example. We take $r=4, s=3, n=5$ and set

$$
A=\left[\begin{array}{ccc}
a & c & e \\
a^{\prime} & d^{\prime} & f \\
b & c^{\prime} & f^{\prime} \\
b^{\prime} & d & e^{\prime}
\end{array}\right], \quad B_{k}=\left[\begin{array}{cc}
g & h \\
g & h^{\prime} \\
g^{\prime} & h \\
g^{\prime} & h^{\prime}
\end{array}\right], k=1,2,3, \quad B_{4}=\left[\begin{array}{cc}
x & y \\
x & y^{\prime} \\
x^{\prime} & z \\
x^{\prime} & z^{\prime}
\end{array}\right] .
$$

Then we have

$$
A \models\left(B_{1}, B_{2}, B_{3}, B_{4}\right)=\left[\begin{array}{ccccc}
a & c & e & g & h \\
a & c & e & g & h^{\prime} \\
a & c & e & g^{\prime} & h \\
a & c & e & g^{\prime} & h^{\prime} \\
a^{\prime} & d^{\prime} & f & g & h \\
a^{\prime} & d^{\prime} & f & g & h^{\prime} \\
a^{\prime} & d^{\prime} & f & g^{\prime} & h \\
a^{\prime} & d^{\prime} & f & g^{\prime} & h^{\prime} \\
b & c^{\prime} & f^{\prime} & g & h \\
b & c^{\prime} & f^{\prime} & g & h^{\prime} \\
b & c^{\prime} & f^{\prime} & g^{\prime} & h \\
b & c^{\prime} & f^{\prime} & g^{\prime} & h^{\prime} \\
b^{\prime} & d & e^{\prime} & x & y \\
b^{\prime} & d & e^{\prime} & x & y^{\prime} \\
b^{\prime} & d & e^{\prime} & x^{\prime} & z \\
b^{\prime} & d & e^{\prime} & x^{\prime} & z^{\prime}
\end{array}\right] .
$$

In this case $A$ and the $B_{k}$ are orthogonal, and so the matrix $A=\left(B_{1}, B_{2}, B_{3}, B_{4}\right)$ is also orthogonal.

Proposition 7 Let $X:=A \models\left(B_{1}, B_{2}, \ldots, B_{r}\right)$ where $A \in \mathcal{O}(r, s), B_{k} \in \mathcal{O}\left(m_{k}, n-s\right)$ for $k=1,2, \ldots, r$. Then $X$ is a UOM if and only if $A$ and all the $B_{k}$ are UOMs.

Proof. Assume that $X$ is a UOM. If $A$ is not a UOM, choose a row $u \in \mathcal{M}(1, s)$ such that $u \perp A$. If $v$ is the first row of $B_{1}$ then row [ $u v$ ] is orthogonal to $X$. Thus we have a contradiction. Similarly, we get a contradiction if at least one $B_{k}$ is not a UOM.

Now assume that $A$ and all the $B_{k}$ are UOMs. Let $[u v] \in \mathcal{M}(1, n)$ with $u \in \mathcal{M}(1, s)$. Since $A$ is a UOM, $u$ is not orthogonal to some row of $A$, say the first row. Since $B_{1}$ is a UOM, $v$ is not orthogonal to some row of $B_{1}$, say the first row. Thus $[u v]$ is not orthogonal to the first row of $X$. Hence, $X$ is not extendible, i.e., $X$ is a UOM.

Corollary 8 If $r \in \Theta_{s}$ and $m_{1}, m_{2}, \ldots, m_{r} \in \Theta_{n-s}$ then $\sum m_{i} \in \Theta_{n}$. 
For example, as $2 \in \Theta_{1}$ and $4,8 \in \Theta_{3}$ we obtain that $8,12 \in \Theta_{4}$.

Two extreme cases of this corollary are used often. The first case is $s=1$, which implies that $r=2$. It has been used extensively by Johnston [17, Proposition 1]. The other extreme case is $s=n-1$, which implies that all $m_{k}=2$.

We say that a matrix $X \in \mathcal{M}(m, n)$ is decomposable if it is equivalent to a matrix $A \models\left(B_{1}, B_{2}, \ldots, B_{r}\right), r \geq 1$.

We exhibit below two UOMs in $\mathcal{O}(8,4)$, the first one is decomposable while the second one is not.

$$
\left[\begin{array}{cccc}
x & a & c & e \\
x & a^{\prime} & d & f \\
x & b & c^{\prime} & f^{\prime} \\
x & b^{\prime} & d^{\prime} & e^{\prime} \\
x^{\prime} & g & i & k \\
x^{\prime} & g^{\prime} & j & l \\
x^{\prime} & h & i^{\prime} & l^{\prime} \\
x^{\prime} & h^{\prime} & j^{\prime} & k^{\prime}
\end{array}\right], \quad\left[\begin{array}{cccc}
a & c & e & g \\
a & d & e^{\prime} & h \\
a & d^{\prime} & f & g^{\prime} \\
a^{\prime} & c^{\prime} & f & h \\
a^{\prime} & c & e & g \\
b & d^{\prime} & f^{\prime} & g^{\prime} \\
b & d & e^{\prime} & h^{\prime} \\
b^{\prime} & c^{\prime} & f^{\prime} & h^{\prime}
\end{array}\right] .
$$
$\mathcal{O}(5)$.

We give below an example of an orthogonal $17 \times 5$ matrix which is orthogonal to 16 rows but has no extensions in

Example 9 Let $Y \in \mathcal{O}(6,4)$ be the UOM given in (2), and $Z$ any UOM in $\mathcal{O}(12,4)$ having no vector variables in common with $Y$. Then the matrix $X:=\left[\begin{array}{c}s \\ s^{\prime}\end{array}\right] \models(Y, Z) \in \mathcal{O}(18,5)$ is a UOM ( $s$ is a new vector variable). Let $P$ be the submatrix of $X$ obtained by removing the first row. So $P=\left[\begin{array}{c}s \\ s^{\prime}\end{array}\right] \models(Q, Z) \in \mathcal{O}(17,5)$ where

$$
Q=\left[\begin{array}{cccc}
x^{\prime} & b & d & e \\
a & y^{\prime} & d^{\prime} & f \\
a^{\prime} & c & z^{\prime} & e^{\prime} \\
a & b^{\prime} & d & w^{\prime} \\
x^{\prime} & c^{\prime} & d^{\prime} & f^{\prime}
\end{array}\right]
$$

There are exactly 16 rows orthogonal to $Q$, say $q_{1}, \ldots, q_{16}$. Let us list these rows:

$$
\begin{aligned}
& \text { [ } \left.\begin{array}{llll}
a^{\prime} & b^{\prime} & z & f
\end{array}\right],\left[\begin{array}{llll}
a^{\prime} & b^{\prime} & d & e
\end{array}\right],\left[\begin{array}{llll}
a^{\prime} & c^{\prime} & d^{\prime} & f
\end{array}\right],\left[\begin{array}{llll}
a^{\prime} & c^{\prime} & d & e^{\prime}
\end{array}\right],
\end{aligned}
$$

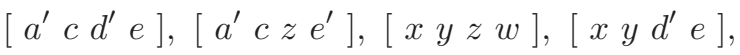

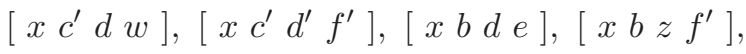

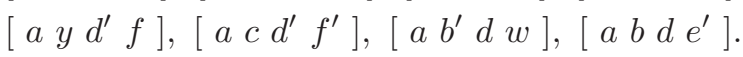

It follows that among the $q_{j}$ s there are at most 11 mutually orthogonal rows. There are also exactly 16 rows orthogonal to $P$, namely the rows $\left[s q_{j}\right]$. Hence, at most 11 of these extended rows may be mutually orthogonal. Since $17+11=28<32, P$ has no extensions in $\mathcal{O}(5)$.

We shall need later the following simple lemma.

Lemma 10 Let $X \in \mathcal{O}(m, n)$ be partitioned as $X=\left[X_{1} X_{2}\right]$, where $X_{1} \in \mathcal{O}(m, s)$. Suppose that $X_{1}$ has a p-extension $Y_{1}$. Then $X$ has a k-extension $Y$ with $k=2^{n-s}(m+p)-m$.

Proof. We may assume that $Y_{1}=\left[\begin{array}{c}X_{1} \\ U\end{array}\right]$. For each $i \in\{1,2, \ldots, m+p\}$ choose a matrix $Z_{i} \in \mathcal{O}(n-s)$. If $i \leq m$ we choose $Z_{i}$ so that its first row is equal to the row $i$ of $X_{2}$. Let $Z$ be the $2^{n-s}(m+p) \times(n-s)$ matrix obtained by stacking the matrices $Z_{1}, Z_{2}, \ldots, Z_{m+p}$ one on top of the other. Let $\tilde{Y}_{1}$ be the $2^{n-s}(m+p) \times s$ matrix obtained from $Y_{1}$ by replacing each row by $2^{n-s}$ copies of it.

We claim that the $2^{n-s}(m+p) \times n$ matrix $Y=\left[\tilde{Y}_{1} Z\right]$ is orthogonal. To prove this claim, let $u:=[a b]$ and $v:=\left[\begin{array}{ll}c & d\end{array}\right]$ be two rows of $Y$, where $a$ and $c$ have length $s$. Since no two rows of $Y$ are equal, we have $a \neq c$ or $b \neq d$. If $a \neq c$ then $a$ and $c$ are orthogonal since they are two rows of $Y_{1}$. If $a=c$ then $b \neq d$ and $b$ and $d$ are orthogonal since they are two rows of some $Z_{i}$. We conclude that $u$ and $v$ are orthogonal and our claim is proved.

Finally, it is easy to verify that $X$ is a submatrix of $Y$.

Note that if $p=2^{s}-m$ then $k=2^{n}-m$, i.e., if $Y_{1} \in \mathcal{O}(s)$ then $Y \in \mathcal{O}(n)$. 
Let us give an example with $m=n=3, s=2, p=1, k=5$ :

$$
X=\left[\begin{array}{ccc}
a & b & c \\
a^{\prime} & d & e \\
a & b^{\prime} & e^{\prime}
\end{array}\right], \quad X_{1}=\left[\begin{array}{cc}
a & b \\
a^{\prime} & d \\
a & b^{\prime}
\end{array}\right], \quad X_{2}=\left[\begin{array}{c}
c \\
e \\
e^{\prime}
\end{array}\right]
$$

Then we have

$$
Y_{1}=\left[\begin{array}{cc}
a & b \\
a^{\prime} & d \\
a & b^{\prime} \\
a^{\prime} & d^{\prime}
\end{array}\right], \quad Z_{1}=\left[\begin{array}{c}
c \\
c^{\prime}
\end{array}\right], \quad Z_{2}=\left[\begin{array}{c}
e \\
e^{\prime}
\end{array}\right], \quad Z_{3}=\left[\begin{array}{c}
e^{\prime} \\
e
\end{array}\right], \quad Z_{4}=\left[\begin{array}{c}
x \\
x^{\prime}
\end{array}\right]
$$

where $x$ could be a new vector variable or one of $c, c^{\prime}, e, e^{\prime}$. Finally,

$$
Y_{1}=\left[\begin{array}{cc}
a & b \\
a & b \\
a^{\prime} & d \\
a^{\prime} & d \\
a & b^{\prime} \\
a & b^{\prime} \\
a & d^{\prime} \\
a & d^{\prime}
\end{array}\right], \quad Y=\left[\begin{array}{ccc}
a & b & c \\
a & b & c^{\prime} \\
a^{\prime} & d & e \\
a^{\prime} & d & e^{\prime} \\
a & b^{\prime} & e^{\prime} \\
a & b^{\prime} & e \\
a & d^{\prime} & x \\
a & d^{\prime} & x^{\prime}
\end{array}\right] .
$$

\section{OLD AND NEW FACTS ABOUT MULTIQUBIT UPBS}

The problem of computing $\Theta_{n}$ has been considered by several authors [1, 3, 11]. In the following theorem we list the main facts presently known about $\Theta_{n}$. For these we refer to the papers of Di Vincenzo et al. [10], Feng [11] and Johnston [16, Theorems 1] and [17, Propositions 2,3 and Theorems 4,5]. The fact that $2^{n}-5 \notin \Theta_{n}$ has been proved recently in $[9$, Theorem 1$]$.

We set $\theta_{n}=\min \Theta_{n}$, the smallest integer of $\Theta_{n}$.

Theorem 11 (i) $\theta_{n}=n+1$ if $n$ is odd; $\theta_{n}=n+2$ if $n \equiv 2(\bmod 4) ; \theta_{n}=n+4$ if $n \equiv 0(\bmod 4)$ and $n>8 ; \theta_{4}=6$ and $\theta_{8}=11$.

(ii) $n+2 \notin \Theta_{n}$ if $n$ is odd.

(iii) $2^{n} \in \Theta_{n}$ for all $n ; 2^{n}-4 \in \Theta_{n}$ for $n \geq 3$; and $2^{n}-k \notin \Theta_{n}$ for all $n$ and $k=1,2,3,5$.

(iv) $m \in \Theta_{n}$ if $n \geq 7$ and $\sum_{k=4}^{n-1} \theta_{k} \leq m \leq 2^{n}-6$.

(v) $m \in \Theta_{n}$ if $n<m \leq 2^{n}$ and $m \equiv 0(\bmod 4)$, except for the case where $n \equiv 1(\bmod 4)$ and $m=2 n+2$ which in general remains undecided.

The sum $\sum_{k=4}^{n-1} \theta_{k}$ is very closely approximated by the quadratic polynomial $n(n+3) / 2-15$. It is shown in [17] that the differences

$$
(n(n+3) / 2-15)-\sum_{k=4}^{n-1} \theta_{k}, \quad n \geq 7,
$$

belong to $\{0,1,2\}$. One can easily verify that for $n \geq 9$ this sequence is periodic with period $0,1,1,2$.

In Table IV we give the UOM of sizes $11 \times 5,10 \times 6,11 \times 6,13 \times 6$ and $m \times 7$ for $m=13,14,15,19$. As far as we know, no such UOM have been discovered so far. Another example of $19 \times 7 \mathrm{UOM}$, not equivalent to the one in this table, can now be constructed by using Corollary 8 . Indeed, we know that $8,11 \in \Theta_{6}$ and so $19 \in \Theta_{7}$. Let us explain the notation used in Table IV] The same lower and upper case letters, say "a" and "A", are perpendiculars of each other, i.e., $A=a^{\prime}$. We write the matrix by listing its rows in order. To save space we omit blanks between the letters in the same row. All entries in column, say $j$, should be adorned with the subscript $j$, so that no variable occurs in two different columns.

From the above theorem we deduce the following simple corollary.

Corollary 12 We have $\Theta_{n} \subseteq \Theta_{n}^{\prime}$ where

(a) $\Theta_{n}^{\prime}=\left\{n+1, n+3, n+4, \ldots, 2^{n}-6,2^{n}-4,2^{n}\right\}$ for $n$ odd;

(b) $\Theta_{n}^{\prime}=\left\{\theta_{n}, \theta_{n}+1, \ldots, 2^{n}-6,2^{n}-4,2^{n}\right\}$ for $n$ even. 
Since it is known that $\Theta_{1}=\{2\}, \Theta_{2}=\{4\}, \Theta_{3}=\{4,8\}$ and $\Theta_{4}=\{6-10,12,16\}$, see [17, Table 3], we have $\Theta_{n}=\Theta_{n}^{\prime}$ for $n \leq 4$. Since we have constructed UOMs of new sizes: $11 \times 5, m \times 6$ for $m \in\{10,11,13\}$, and $m \times 8$ for $m \in\{12-15,17-19\}$ (see Sec. IX Table IV), we have also $\Theta_{5}=\Theta_{5}^{\prime}, \Theta_{6}=\Theta_{6}^{\prime}$ and $\Theta_{8}=\Theta_{8}^{\prime}$. (The abbreviation $i-j$ stands for the sequence $i, i+1, \ldots, j$ of all integers in the range from $i$ to $j$.) We were not able to decide whether 10 or 11 belongs to $\Theta_{7}$.

For other $n \leq 13$, we list in Table 1 the integers known to belong to $\Theta_{n}$.

\begin{tabular}{cl}
$n$ & \multicolumn{1}{c}{$m$} \\
\hline & \multicolumn{1}{c}{} \\
7 & $8,12-122,124,128$ \\
9 & $10,12,16,22-506,508,512$ \\
10 & $12,16,20-22,24,26,28,32-1018,1020,1024$ \\
11 & $12,16,20,24,28,32,34,36,38,40,42,44-2042,2044,2048$ \\
12 & $16,20,24,28,32,36,40,42,44,46,48,50-4090,4092,4096$ \\
13 & $14,16,20,24,32,36,40,44,48,52,56,58,60-8186,8188,8192$
\end{tabular}

Table I: Known cases of $m \in \Theta_{n}, n=7,9-13$.

Very little is known about the number of UOM equivalence classes in $\mathcal{O}(m, n), m \in \Theta_{n}$. In Table $\llbracket$ we record the known facts for $m \leq 12$. (The blanks occur outside the range $\theta_{n} \leq m \leq 2^{n}$.) The main reference is Johnston's paper [17] where the electronic links to his computational results are provided.

\begin{tabular}{|c|c|c|c|c|c|c|c|c|c|c|}
\hline$m \backslash n$ & 12 & 3 & 4 & 5 & 6 & 7 & 8 & 9 & 10 & 11 \\
\hline 1 & & & & & & & & & & \\
\hline 2 & 1 & & & & & & & & & \\
\hline 3 & & & & & & & & & & \\
\hline 4 & 2 & 1 & & & & & & & & \\
\hline 5 & & 0 & & & & & & & & \\
\hline 6 & & 0 & 1 & 1 & & & & & & \\
\hline 7 & & 0 & 1 & 0 & & & & & & \\
\hline 8 & & 17 & 144 & 32 & 9 & 6 & & & & \\
\hline 9 & & & 11 & $e$ & $e$ & 0 & & & & \\
\hline 10 & & & 80 & $e$ & $e$ & $?$ & & $e$ & & \\
\hline 11 & & & 0 & $e$ & $e$ & $?$ & $e$ & 0 & & \\
\hline 12 & & & 1209 & $e$ & $e$ & $e$ & $e$ & $e$ & $e$ & $e$ \\
\hline
\end{tabular}

Table II: Number of UOM equivalence classes. The letter $e$ means that the UOMs exist in $\mathcal{O}(m, n)$. The two question marks indicate that the existence of UOMs is still undecided.

For instance, there is only 1 UOM-equivalence class in $\mathcal{O}(2,1)$, only 2 UOM-equivalence classes in $\mathcal{O}(4,2)$, and only 1 UOM-equivalence class in $\mathcal{O}(4,3)$. The two non-equivalent UOMs in $\mathcal{O}(2)$ are exhibited in (1).

Most of these results have been obtained by computer searches. Some are easy to verify. For instance let us verify the claim for $(m, n)=(4,3)$. Let $X=\left[x_{i, j}\right] \in \mathcal{O}(4,3)$. By Lemma 5 (ii) we have $\mu(X)=1$, i.e. $\mu\left(x_{i, j}\right)=1$ for all $i, j$. By using Lemma 5 (i), we deduce that each column of $X$ must contain exactly two independent vector variables and their perpendiculars. By permuting the rows of $X$, we can assume that $x_{2,1}=x_{1,1}^{\prime}, x_{4,1}=x_{3,1}^{\prime}$ and $x_{1,2}=x_{3,2}^{\prime}$. It follows that $x_{2,2}=x_{4,2}^{\prime}$. Since the first and fourth rows are orthogonal, we have $x_{1,4}=x_{4,4}^{\prime}$. Hence, we must have $x_{2,4}=x_{3,4}^{\prime}$ and so $X$ is equivalent to the first matrix in (2).

Up to column permutations, a matrix $X=\left[x_{i j}\right] \in \mathcal{O}(m, n)$ can be replaced by the set $\left\{X_{1}, X_{2}, \ldots, X_{n}\right\}$, where the temporary symbol $X_{r}$ represents the column $r$ of $X$. Let $k$ be the number of independent vector variables in column $r$, i.e., $k=\nu_{r}=\nu_{r}(X)$. We choose a maximal independent set $\left\{a_{1}, a_{2}, \ldots, a_{k}\right\}$ of vector variables in column $r$. For each $s \in\{1,2, \ldots, k\}$ we define the index sets $\Omega_{s, r}^{\prime}=\left\{i: x_{i r}=a_{s}\right\}$ and $\Omega_{s, r}^{\prime \prime}=\left\{i: x_{i r}=a_{s}^{\prime}\right\}$, and we set 
$\Omega_{s, r}=\left\{\Omega_{s, r}^{\prime}, \Omega_{s, r}^{\prime \prime}\right\}$. Finally we replace the temporary symbol $X_{r}$ with the set $\Omega_{r}:=\left\{\Omega_{1, r}, \ldots, \Omega_{k, r}\right\}$. We shall refer to the set $\Omega:=\left\{\Omega_{1}, \ldots, \Omega_{n}\right\}$ as the symbol of $X$.

For instance, for the first matrix in (4) we have

$$
\Omega=\left\{\left\{\Omega_{1,1}, \Omega_{2,1}\right\},\left\{\Omega_{1,2}, \Omega_{2,2}\right\},\left\{\Omega_{1,3}, \Omega_{2,3}\right\},\left\{\Omega_{1,4}, \Omega_{2,4}, \Omega_{3,4}\right\}\right\},
$$

where

$$
\begin{aligned}
& \Omega_{1,1}=\{\{1,2,3\},\{4,5\}\}, \quad \Omega_{2,1}=\{\{6\},\{7\}\}, \\
& \Omega_{1,2}=\{\{1,4\},\{2,6\}\}, \quad \Omega_{2,2}=\{\{3,5\},\{7\}\}, \\
& \Omega_{1,3}=\{\{1,4\},\{7\}\}, \quad \Omega_{2,3}=\{\{2,5\},\{3,6\}\}, \\
& \Omega_{1,4}=\{\{1,6\},\{3\}\}, \quad \Omega_{2,4}=\{\{2\},\{7\}\}, \quad \Omega_{3,4}=\{\{4\},\{5\}\} .
\end{aligned}
$$

Since we assumed only that $X \in \mathcal{O}(m, n)$, it may happen that some of the index sets $\Omega_{s, r}^{\prime \prime}$ are empty. However, this is not the case if $X$ is an UOM. Note that the symbol $\Omega$ is uniquely determined by $X$. It does not depend on the choice of the maximal sets $\left\{a_{1}, a_{2}, \ldots, a_{k}\right\}$ of independent vector variables in a column. It also does not depend on the ordering of these variables. Moreover, it is affected by neither column permutations nor renaming of the vector variables. The row permutations of $X$ do change the symbol $\Omega$. Their effect consists in permuting the integers $1,2, \ldots, m$. More precisely, if $\pi$ is a permutation of these integers, then $\pi \Omega$ is obtained from $\Omega$ by simultaneously replacing each integer $i$ by $\pi(i)$ (all $n$ occurencies of $i$ in $\Omega$ ).

We can recover $X$ up to equivalence from its symbol $\Omega$. To do this, for $r \in\{1,2, \ldots, n\}$ let $k$ be the cardinality of the set $\Omega_{r}:=\left\{\Omega_{1, r}, \ldots, \Omega_{k, r}\right\}$. We select a set of independent vector variables $\left\{z_{1}, z_{2}, \ldots, z_{k}\right\}$ and define the column $r$ of the matrix $Y=\left[y_{i j}\right] \in \mathcal{M}(m, n)$ by setting $y_{i r}=z_{s}$ if $i \in \Omega_{s, r}^{\prime}$ and $y_{i r}=z_{s}^{\prime}$ if $i \in \Omega_{s, r}^{\prime \prime}$. Then the matrices $X$ and $Y$ are equivalent. (The two choices for the ordered pair $\left(\Omega_{s, r}^{\prime}, \Omega_{s, r}^{\prime \prime}\right)$ give equivalent matrices $Y$.)

Thus we obtain the following simple test for equivalence of two UOMs.

Lemma 13 Two UOMs $X$ and $Y$ in $\mathcal{O}(m, n)$ with symbols $\Omega_{X}$ and $\Omega_{Y}$, respectively, are equivalent if and only if $\Omega_{Y}=\pi \Omega_{X}$ for some permutation $\pi$ of $\{1,2, \ldots, m\}$.

(Note that this test is not efficient for large values of $n$.)

\section{NEW CONSTRUCTION OF UOM}

Let $X \in \mathcal{M}(2 m, n)$ be partitioned into two blocks, $X_{1}$ and $X_{2}$, of the same size, and assume that $X_{1}, X_{2} \in \mathcal{O}(m, n)$. From $X$ we can construct a matrix $Z \in \mathcal{O}(2 m, m+n)$.

As the first step of our construction, we choose a matrix $Y_{1}:=\left[y_{i j}\right]$ of order $m$ where $y_{i j}$ are new vector variables which are mutually independent and also independent from the varables that occur in $X$.

The second step is to construct another matrix of order $m$. This matrix, $Y_{2}$, is obtained from $Y_{1}$ by first replacing each entry by its perpendicular and then, for each $j \in\{1,2, \ldots, m\}$, rotating the entries in the $j$ th column $j-1$ steps downwards.

For example, if $m=4$ we have

$$
Y_{1}=\left[\begin{array}{llll}
y_{11} & y_{12} & y_{13} & y_{14} \\
y_{21} & y_{22} & y_{23} & y_{24} \\
y_{31} & y_{32} & y_{33} & y_{34} \\
y_{41} & y_{42} & y_{43} & y_{44}
\end{array}\right], \quad Y_{2}=\left[\begin{array}{llll}
y_{11}^{\prime} & y_{42}^{\prime} & y_{33}^{\prime} & y_{24}^{\prime} \\
y_{21}^{\prime} & y_{12}^{\prime} & y_{43}^{\prime} & y_{34}^{\prime} \\
y_{31}^{\prime} & y_{22}^{\prime} & y_{13}^{\prime} & y_{44}^{\prime} \\
y_{41}^{\prime} & y_{32}^{\prime} & y_{23}^{\prime} & y_{14}^{\prime}
\end{array}\right] .
$$

From the definition of $Y_{2}$ it follows that for each $i$ no two of the vector variables $y_{i, 1}^{\prime}, y_{i, 2}^{\prime}, \ldots, y_{i, m}^{\prime}$ occur in the same row of $Y_{2}$. Thus each row of $Y_{1}$ is perpendicular to each row of $Y_{2}$. This property of the matrices $Y_{1}$ and $Y_{2}$ is essential for our construction.

Finally we define our matrix $Z$ by setting

$$
Z=\left[\begin{array}{ll}
X_{1} & Y_{1} \\
X_{2} & Y_{2}
\end{array}\right]
$$

The fact that $Z \in \mathcal{O}(2 m, m+n)$ follows immediately from the above mentioned property and the hypothesis that $X_{1}, X_{2} \in \mathcal{O}(m, n)$. 
For the sake of brevity, we shall write $X \Longrightarrow Z$ or $\left(X_{1}, X_{2}\right) \Longrightarrow Z$ to indicate that $Z$ is obtained from $X$ by the above construction, and by choosing the matrix $Y_{1}$ appropriately.

Let us assume now that $n$ is odd. In that case $\theta_{n}=n+1$. The $n$-qubit UPBs of cardinality $n+1$, known as GenShift UPBs, have been constructed in [10, p. 395]. They give UOMs in $\mathcal{O}(n+1, n)$, which will be called GenShift UOMs.

Let us recall that construction. Let $n=2 p+1$ and denote by $Z$ the $n+1$ by $n$ matrix obtained from the cyclic

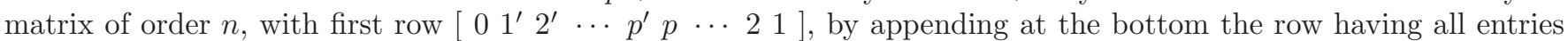
equal to $0^{\prime}$. Next for each $j \in\{1,2, \ldots, n\}$ choose $p+1$ independent vector variables $y_{i, j}, i=0,1, \ldots, p$. Finally, construct $X$ from $Z$ by replacing, for each $j$, the entries in column $j$ by vector variables as follows: $k \rightarrow y_{k, j}$ and $k^{\prime} \rightarrow y_{k, j}^{\prime}, k \in\{0,1, \ldots, p\}$.

Obviously, the last row of $X$ is orthogonal to all other rows. Let us verify that the rows $i$ and $j, 1 \leq i<j \leq n$, of $X$ are orthogonal. If $j-i$ is even, this follows from $Z_{i, i+k}=k^{\prime}$ and $Z_{j, j-k}=k$, where $k=(j-i) / 2$. If $j-i$ is odd, it follows from $Z_{i, i-k}=k$ and $Z_{j, j+k}=k^{\prime}$, where $k=p-(j-i-1) / 2$. (The second subscript should be reduced modulo $n$ to be in the range $1,2, \ldots, n$.) As no column of $Z$ has two equal entries, Corollary 6 implies that $X$ is an UOM.

Let us give an example. We take $n=5$, and choose 15 independent vector variables $y_{i, j}, i=0,1,2 ; j=1, \ldots, 5$. Then the matrix $Z$ and the corresponding matrix $X$ are

$$
Z=\left[\begin{array}{ccccc}
0 & 1^{\prime} & 2^{\prime} & 2 & 1 \\
1 & 0 & 1^{\prime} & 2^{\prime} & 2 \\
2 & 1 & 0 & 1^{\prime} & 2^{\prime} \\
2^{\prime} & 2 & 1 & 0 & 1^{\prime} \\
1^{\prime} & 2^{\prime} & 2 & 1 & 0 \\
0^{\prime} & 0^{\prime} & 0^{\prime} & 0^{\prime} & 0^{\prime}
\end{array}\right], \quad X=\left[\begin{array}{lllll}
y_{01} & y_{12}^{\prime} & y_{23}^{\prime} & y_{24} & y_{15} \\
y_{11} & y_{02} & y_{13}^{\prime} & y_{24}^{\prime} & y_{25} \\
y_{21} & y_{12} & y_{03} & y_{14}^{\prime} & y_{25}^{\prime} \\
y_{21}^{\prime} & y_{22} & y_{13} & y_{04} & y_{15}^{\prime} \\
y_{11}^{\prime} & y_{22}^{\prime} & y_{23} & y_{14} & y_{05} \\
y_{01}^{\prime} & y_{02}^{\prime} & y_{03}^{\prime} & y_{04}^{\prime} & y_{05}^{\prime}
\end{array}\right]
$$

We present now our alternative construction of UOMs in $\mathcal{O}(n+1, n)$ when $n=2 p+1$ and $p$ is odd. Since $\theta_{p}=p+1$, there exist UOMs in $\mathcal{O}(p+1, p)$ e.g. the above GenShift UOM.

Proposition 14 Let $X_{1}, X_{2} \in \mathcal{O}(p+1, p)$ be UOMs having no vector variable in common and let

$$
\left(X_{1}, X_{2}\right) \Longrightarrow Z=\left[\begin{array}{ll}
X_{1} & Y_{1} \\
X_{2} & Y_{2}
\end{array}\right] \in \mathcal{O}(2 p+2,2 p+1) \text {. }
$$

Then the matrix $Z$ is a UOM.

Proof. From the construction of $Z$ we know that $Z$ is an orthogonal matrix. It is easy to see that $\mu(Z)=1$. Hence, $Z$ is a UOM by Corollary 6 .

This construction can be generalized by using different matrices $Y_{2}$. Let $\pi_{1}, \pi_{2}, \ldots, \pi_{p+1}$ be permutations of the set $\{1,2, \ldots, p+1\}$ such that each permutation $\pi_{j}^{-1} \pi_{k}, j \neq k$, is fixed-point-free, i.e., $\pi_{j}^{-1} \pi_{k}(i) \neq i$ for all $i$. Then we can take $Y_{2}$ to be the matrix whose $(i, j)$ th entry is $y_{\pi_{j}(i), j}^{\prime}$. The row $i$ of $Y_{2}$ is

$$
\left[\begin{array}{llll}
y_{\pi_{1}(i), 1}^{\prime} & y_{\pi_{2}(i), 2}^{\prime} & \cdots & y_{\pi_{p+1}(i), p+1}^{\prime}
\end{array}\right]
$$

Since $\pi_{j}^{-1} \pi_{k}(i) \neq i$ whenever $j \neq k$, we have $\left\{\pi_{j}(i): j=1,2, \ldots, p+1\right\}=\{1,2, \ldots, p+1\}$. We infer that the row $i$ of $Y_{2}$ is orthogonal to $Y_{1}$. Since $i$ is arbitrary, we have $Y_{1} \perp Y_{2}$.

There are many choices for the permutations $\pi_{j}$ that satisfy the condition stated above. For instance, we can choose any $(p+1)$-cycle $\sigma$ and set $\pi_{j}=\sigma^{j-1}$ for $j \in\{1,2, \ldots, p+1\}$. If $p=3$ and we choose $\sigma=(1432)$ then the matrix $Y_{2}$ is exactly the one shown in (6). There are other choices as well. For instance, for $p=3$ we can take $\pi_{1}=i d$, $\pi_{2}=(12)(34), \pi_{3}=(13)(24)$ and $\pi_{4}=(14)(23)$, the elements of the Klein four-group. Then we obtain that

$$
Y_{2}=\left[\begin{array}{llll}
y_{11}^{\prime} & y_{22}^{\prime} & y_{33}^{\prime} & y_{44}^{\prime} \\
y_{21}^{\prime} & y_{12}^{\prime} & y_{43}^{\prime} & y_{34}^{\prime} \\
y_{31}^{\prime} & y_{42}^{\prime} & y_{13}^{\prime} & y_{24}^{\prime} \\
y_{41}^{\prime} & y_{32}^{\prime} & y_{23}^{\prime} & y_{14}^{\prime}
\end{array}\right] .
$$

Let us apply our construction in the case $n=7$. There are 6 UOM-equivalence classes in $\mathcal{O}(8,7)$. They are listed on Johnston's website and we denote them as $J_{i}, i=1, \ldots, 6$. We list them here for the reader's convenience: 


$$
\begin{aligned}
& J_{1}=[a a a a a a a, A b b b b b b, b A B c c c c, B B A d d d d, c c c A B C D, C d d B A D C, d C D C D A B, D D C D C B A], \\
& J_{2}=[a a a a a a a, A b b b b b b, b A B c c c c, B c A B d d d, c d c A B C D, C C d d A B C, d D D C D A B, D B C D C D A], \\
& J_{3}=[a a a a a a a, A b b b b b b, b A c c B c c, B c A B c d d, c d C A d B D, C C d d A C B, d D B D C A C, D B D C D D A], \\
& J_{4}=[a a a a a a a, A b b b b b b, b A c c c B c, B c A B d c d, c d d A B C C, C C C d A d B, d D B D C A D, D B D C D D A], \\
& J_{5}=[a a a a a a a, A b b b b b b, b A c c c B c, B c A d d c B, c d d A B C C, C C C B A d d, d D B C D A D, D B D D C D A], \\
& J_{6}=[a a a a a a a, A b b b b b b, b A c c c c B, B c A d d B c, c d d A B C C, C C C B A d d, d D B C D A D, D B D D C D A] .
\end{aligned}
$$

For $k=1,2$ we set

$$
X_{k}=\left[\begin{array}{ccc}
a_{k} & b_{k} & c_{k} \\
a_{k}^{\prime} & e_{k}^{\prime} & f_{k} \\
d_{k} & b_{k}^{\prime} & f_{k}^{\prime} \\
d_{k}^{\prime} & e_{k} & c_{k}^{\prime}
\end{array}\right] .
$$

For $Y_{1}$ we take the first matrix given in (6) while for $Y_{2}$ we use four choices for the permutations $\left\{\pi_{i}\right\}$ : the above Klein four-group $K$, its coset $(1,2,3) K$, the cyclic group $C$ generated by $(1,2,3,4)$, and its coset $(1,2) C$. (The ordering of the $\pi_{i}$ is irelevant in these cases.) By using our equivalence test (see Lemma 13) we have verified that the four UOMs obtained in this way are equivalent to $J_{1}, J_{2}, J_{6}$ and $J_{5}$, respectively. GenShift UOM is equivalent to $J_{3}$.

Our construction can be used to generate many UOMs of different sizes. Let us give a few examples. In these examples we use our construction $\left(X_{1}, X_{2}\right) \Longrightarrow Z$ by specifying $X_{1}$ to be an orthogonal matrix of size $m \times n$ while $X_{2}$ will always be obtained from $X_{1}$ by renaming the vector variables. Thus instead of $\left(X_{1}, X_{2}\right) \Longrightarrow Z$ we shall write just $X_{1} \Longrightarrow Z$. The auxilliary matrix $Y_{1}$ from our construction does not play an important role and we may consider it as being fixed. In this way we obtain a map $\mathcal{O}(m, n) \rightarrow \mathcal{O}(2 m, m+n)$. Note that even when $X_{1}$ is an UOM, $Z$ is not necessarily an UOM.

For each positive odd integer $n$ we obtain an infinite sequence of maps:

$$
\mathcal{O}(n+1, n) \rightarrow \mathcal{O}(2 n+2,2 n+1) \rightarrow \mathcal{O}(4 n+4,4 n+3) \rightarrow \cdots
$$

Each of the maps in this sequence preserves UOMs in the sense that the image of a UOM is again a UOM. This follows from Proposition [14. By taking $n=1$, from the trivial UOM in $\mathcal{O}(1)=\mathcal{O}(2,1)$ we obtain UOMs in $\mathcal{O}(4,3)$, $\mathcal{O}(8,7)$, etc.

For each positive integer $n \equiv 2(\bmod 4)$ there is another infinite sequence of UOMs:

$$
X_{n} \Longrightarrow X_{2 n+2} \Longrightarrow X_{4 n+6} \Longrightarrow \cdots
$$

with $X_{k} \in \mathcal{O}(k+2, k)$ for $k=n, 2 n+2,4 n+6, \ldots$ It is essential here that $X_{n}$ be chosen so that $\mu(x)=2$ for all entries $x$ in the first column, which implies that $\mu(x)=1$ for all other entries $x$ of $X_{n}$. It follows from the proof of [11, Theorem 3.2] that such $X_{n}$ exists when $n \equiv 2(\bmod 4)$.

For example, we can set $n=2$ and choose $X_{2}$ to be the third matrix in (11). Then by applying our construction, we obtain UOMs in $\mathcal{O}(8,6), \mathcal{O}(16,14)$, etc.

\section{CONSTRUCTION OF MULTIQUBIT PPT ENTANGLED STATES}

Let $\mathcal{S} \subset \mathcal{H}$ be an OPS. We say that the orthogonal projector $\rho$ onto the subspace $\mathcal{S}^{\perp} \subseteq \mathcal{H}$ is associated with $\mathcal{S}$. If $\Gamma$ is a partial transposition operator, then $\mathcal{S}^{\Gamma}$ is also an OPS and $\rho^{\Gamma}$ is the projector associated with it. This implies that $\rho$ is a PPT state. In the case when $\mathcal{S}$ is a UPB, then $\rho$ is a (non-normalized) PPT entangled state (PPTES). This fact is also valid in arbitrary multipartite systems [2, 10].

Even when $\mathcal{S}$ is not a UPB, $\rho$ may be a PPTES. Such OPS can be constructed from UOMs. The following lemma plays a crucial role in our construction.

Lemma 15 Let $X=\left[x_{i, j}\right] \in \mathcal{O}(m, n)$ be a $U O M$ and let $Y$ be a submatrix of $X$ obtained by removing one of its rows. Then there exist only finitely many rows of vector variables which are orthogonal to $Y$. 
Proof. We may assume that the first row of $X$ has been removed. Let $y:=\left[\begin{array}{llll}y_{1} & y_{2} & \cdots & y_{n}\end{array}\right]$ be a row orthogonal to $Y$. If $y_{1}^{\prime} \notin\left\{x_{2,1}, x_{3,1}, \ldots, x_{m, 1}\right\}$ then the row $\left[x_{1,1}^{\prime} y_{2} \cdots y_{n}\right]$ is orthogonal to $X$. As $X$ is a UOM, we have a contradiction. We conclude that $y_{1} \in\left\{x_{2,1}^{\prime}, x_{3,1}^{\prime}, \ldots, x_{m, 1}^{\prime}\right\}$. Similarly, we must have $y_{j} \in\left\{x_{2, j}^{\prime}, x_{3, j}^{\prime}, \ldots, x_{m, j}^{\prime}\right\}$ for all $j$, and the lemma is proved.

Corollary 16 Let $X$ and $Y$ be as in the above lemma. Further, let $Z$ be the matrix obtained by appending to $Y$ all rows $u_{1}, u_{2}, \ldots, u_{s}$ orthogonal to $Y$. If $\alpha$ is a generic evaluation of $Z$, then the range of the projector $\rho$ associated with the OPS of $\alpha(Y)$ contains only s product vectors, namely $\alpha\left(u_{1}\right), \alpha\left(u_{2}\right), \ldots, \alpha\left(u_{s}\right)$.

Let $X$ and $Y$ be as in Lemma 15. It is very easy to write a computer program which outputs all rows orthogonal to $Y$. Consequently, one can easily compute the product vectors in $\mathcal{R}(\rho)$ in Corollary 16.

If a projector is associated with a UPB, then it is a PPTES of the very special kind because its range contains no product vectors. Note that $s \geq 1$ in Corollary 16. If also $s \leq 2^{n}-m$ then the projector $\rho$ is a PPTES whose range contains $s \geq 1$ product vectors. Hence, these PPTES are never equivalent (under local unitary transformations and qubit permutations) to those associated with the UPBs.

Let $X \in \mathcal{O}(m, n), m<2^{n}$. If $\alpha$ is a generic evaluation of $X$ then, by abuse of language, we say that the projector associated with the OPS of $\alpha(X)$ is also associated with $X$. Now assume that $X$ is an UOM. Then the projectors associated with $X$ are non-normalized PPTES and we say that they are the primary PPTES of $X$. Let us also introduce the secondary PPTES of $X$. These are the entangled projectors which are associated with the $(m-1) \times n$ submatrices $Y$ of $X$. Note that we have here singled out only the projectors associated with the $Y$ s which are entangled. In general, a projector associated with $Y$ does not have to be entangled. For instance, when $n=3$ there are no secondary PPTES.

The secondary PPTES occur first for $n=4$. In the following table, for each $m \in \Theta_{4} \backslash\{16\}$, we list all pairs $(\operatorname{rank} \rho, s)$ where $\rho$ is a secondary PPTES of some $\operatorname{UOM} X \in \mathcal{O}(m, 4)$ and $s$ is the number of product vectors in $\mathcal{R}(\rho)$.

\begin{tabular}{ccc}
$m$ & $\operatorname{rank} \rho$ & $s$ \\
\hline & & \\
6 & 11 & 10 \\
7 & 10 & $3,6,7,8$ \\
8 & 9 & $1,2,3,4,6$ \\
9 & 8 & $1,2,3,4,6$ \\
10 & 7 & $1,2,4$ \\
12 & 5 & 1
\end{tabular}

Table III: Secondary PPTES of four qubits

We point out that the rank-5 PPTES for $m=12$ (see the last line of Table III) support our conjecture in [9, Conjecture 10]. In the case $m=6$ there is only one UOM up to equivalence. We may assume that this is the matrix $Y$ in (2). If we delete the first or fourth row of $Y$ then the projector $\rho$ is separable. In the other four cases it is entangled of rank 11 and has 10 product vectors in its range.

Let us give yet another example. Consider the following $\mathrm{UOM}$ in $\mathcal{O}(7,4)$

$$
X=\left[\begin{array}{cccc}
a & c & e & g \\
a & c^{\prime} & f & h \\
a & d & f^{\prime} & g^{\prime} \\
a^{\prime} & c & e & i \\
a^{\prime} & d & f & i^{\prime} \\
b & c^{\prime} & f^{\prime} & g \\
b^{\prime} & d^{\prime} & e^{\prime} & h^{\prime}
\end{array}\right]
$$

Drop the first row, $u$, from $X$ to get $Y$. Apart from $u$ there are only two other rows orthogonal to $Y$, namely $v:=[a c f h]$ and $w:=\left[a d f h^{\prime}\right]$. Since in this case $v$ and $w$ are orthogonal, if we append $v$ and $w$ to $Y$ we obtain an UOM $Z \in \mathcal{O}(8,4)$. Let $\alpha$ be a generic evaluation of $Z$ and $\mathcal{S}$ the OPS of $\alpha(Y)$. If $\rho$ is the projector associated with $\mathcal{S}$, then $\mathcal{R}(\rho)$ contains only three product vectors, namely $\alpha(u), \alpha(v)$ and $\alpha(w)$. As $\rho$ has rank 10 , it follows that $\rho$ is entangled. Hence, $\rho$ is a PPTES. 
We can also construct PPTES by dropping more than one row from a UOM. We give an example. Let $X$ be the $10 \times 5$ UOM with rows:

$$
\text { [baBCc, cbaBC, CcbaB, BCcba, AAAAA, CaCcb, aACbb, cBbBa, CABBa, caCbC]. }
$$

Let $Y \in \mathcal{O}(8,5)$ be the matrix obtained by dropping the last two rows of $X$. Then there are exactly 6 rows orthogonal to $Y$, namely the two last rows of $X$ and the following four: $a A B B c, c a A c A, c a b b A, C A a B b$. The projector associated with $Y$ has rank 24 and has only 6 product vectors in the range. Hence it is a PPTES.

The construction of PPTES described above for multiqubit systems can be generalized to arbitrary finitedimensional quantum systems, see Proposition 19 in Sec. [X]

\section{A PARTIAL ORDER IN $\mathcal{M}(m, n)$}

Let $X \in \mathcal{M}(m, n)$ and let $x, y$ be two independent vector variables which occur in the same column of $X$, say column $j$. Denote by $Y$ the matrix obtained from $X$ by replacing all occurrencies of $y$ and $y^{\prime}$ in $X$ as follows: $y$ by $x$ and $y^{\prime}$ by $x^{\prime}$ or $y$ by $x^{\prime}$ and $y^{\prime}$ by $x$. Then we shall write $Y \prec X$. Note that we have $\nu_{j}(Y)=\nu_{j}(X)-1$, i.e., the number of independent variables of $Y$ is one less than that of $X$.

The relation " $\prec$ " induces the partial order " $\leq$ " on $\mathcal{M}(m, n)$, known as the transitive closure of " $\prec$ ". Explicitly, for two matrices $X, Y \in \mathcal{M}(m, n)$, we say that $Y \leq X$ if there exists a finite chain

$$
Y=Z_{0} \prec Z_{1} \prec \cdots \prec Z_{k}=X, \quad k \geq 0 .
$$

Further, we write $Y<X$ if $Y \leq X$ and $Y \neq X$.

Assume that $Y \prec X$. Then it is easy to see that $X \in \mathcal{O}(m, n)$ implies that $Y \in \mathcal{O}(m, n)$. However, the converse is false. Further, if $X$ is a UOM then $Y$ is not necessarily a UOM. For instance, if we replace $f$ and $f^{\prime}$ with $e$ and $e^{\prime}$ in the UOM $X \in \mathcal{O}(4,3)$ in (2) then the resulting matrix is not a UOM.

Consequently, if in the chain (11) we have $X \in \mathcal{O}(m, n)$ then all $Z_{i} \in \mathcal{O}(m, n)$.

If $X$ and $Y$ in (11) are UOM then so are all the $Z_{i}$. This follows from the following lemma.

Lemma 17 If $Y \prec X \in \mathcal{O}(m, n)$ and $Y$ is a UOM then $X$ is a $U O M$.

Proof. By the hypothesis, we may assume that $Y$ is obtained from $X$ by identifying two independent variables $a$ and $b$ in the first column of $X$. Say, we replaced each occurrence of $b$ with $a$ (and $b^{\prime}$ with $a^{\prime}$ ).

Suppose that there exists a row of vector variables $u:=\left[\begin{array}{llll}u_{1} & u_{2} & \cdots & u_{n}\end{array}\right]$ which is orthogonal to $X$. Since $Y$ is a UOM, we may assume that $u$ is not orthogonal to the first row $y:=\left[\begin{array}{llll}y_{1} & y_{2} & \cdots & y_{n}\end{array}\right]$ of $Y$. Hence, the first row $x:=\left[\begin{array}{llll}x_{1} & x_{2} & \cdots & x_{n}\end{array}\right]$ of $X$ is not equal to $y$. It follows that $x_{1} \in\left\{b, b^{\prime}\right\}$ and we may assume that $x_{1}=b$, and so $y_{1}=a$. It is now easy to verify that the row $v:=\left[\begin{array}{llll}a^{\prime} & u_{2} & \cdots & u_{n}\end{array}\right]$ is orthogonal to $Y$, which gives a contradiction. Thus we have shown that $X$ has no 1-extensions, i.e., it is a UOM.

We say that a $\mathrm{UOM} X \in \mathcal{O}(m, n)$ is maximal if there is no UOM $Y \in \mathcal{O}(m, n)$ such that $X<Y$. Similarly, we say that a $\mathrm{UOM} X \in \mathcal{O}(m, n)$ is minimal if there is no $\mathrm{UOM} Y \in \mathcal{O}(m, n)$ such that $Y<X$. Further we say that an UOM is isolated if it is both minimal and maximal. In order to prove that a UOM $X$ is maximal, by the above lemma, it suffices to verify that there is no UOM $Y \in \mathcal{O}(m, n)$ such that $X \prec Y$. Similarly, in order to prove that a UOM $X$ is minimal, it suffices to verify that there is no UOM $Y \in \mathcal{O}(m, n)$ such that $Y \prec X$.

These definitions extend naturally to equivalence classes of orthogonal matrices and UOMs. For instance, if $\mathcal{X}$ and $\mathcal{Y}$ are two equivalence classes of matrices in $\mathcal{O}(m, n)$ and $Y \leq X$ for some $X \in \mathcal{X}$ and some $Y \in \mathcal{Y}$, then we shall write $\mathcal{Y} \leq \mathcal{X}$. We say that an equivalence class of UOMs $\mathcal{X} \subseteq \mathcal{O}(m, n)$ is maximal if there is no equivalence class of UOMs $\mathcal{Y} \subseteq \mathcal{O}(m, n)$ such that $\mathcal{X}<\mathcal{Y}$, etc.

Finally, we say that a UOM $X$ and its equivalence class $[X]$ are irreducible if all $\nu_{j}(X)>1$. Otherwise, we say that $X$ and $[X]$ are reducible. Any reducible $\operatorname{UOM} X$ is equivalent to one of the form $\left[\begin{array}{c}a \\ a^{\prime}\end{array}\right] \models(U, V)$, where $U$ and $V$ are UOMs. Thus all reducible UOMs are decomposable.

Lemma 18 Let $X$ be a decomposable $U O M$, say $X=A \models\left(B_{1}, \ldots, B_{r}\right)$. Then

(i) $X$ is maximal if and only if $A$ and all $B_{i}$ are maximal and no two of the blocks $B_{i}$ have a vector variable in common;

(ii) if $X$ is not maximal then there exists a $U O M Y$ such that $X \prec Y$ and $Y$ is obtained from $X$ by modifying a single column in either $A$ or just one of the blocks $B_{i}$. 
Proof. (i) Assume $X$ is maximal. By Proposition 7, $A$ and all $B_{i}$ are UOMs. Assume that some $B_{i}$ is not maximal. Then $B_{i} \prec U$ for some UOM $U$. Hence, $B_{i}$ and $U$ differ only in one column and $U$ has one new independent variable, say $u$. We may assume that $u$ is independent from the variables which occur in $X$. Let $Y$ be the UOM obtained from $X$ by replacing $B_{i}$ with $U$. Then $X \prec Y$ and we have a contradiction since $X$ is maximal. We conclude that each $B_{i}$ must be maximal. Similarly, one can show that $A$ has to be maximal. It remains to consider the case where $A$ and all the $B_{i}$ are maximal. Assume now that one of the vector variables, say $u$, occurs in $B_{i}$ and $B_{j}$ (necessarily in the same column). We choose a new vector variable $v$ which does not occur in $X$. Let $Y$ be the matrix we obtain by replacing all occurrences of $u$ and $u^{\prime}$ in $B_{j}$ with $v$ and $v^{\prime}$, respectively. Then $X \prec Y$ and we have again a contradiction. Hence our assumption must be false, i.e., no vector variable can occur in two blocks $B_{i}$ and $B_{j}$. This completes the proof of the "if" part.

The "only if" part follows immediately from the definition of " $\prec "$.

(ii) This follows from the proof of (i) by observing that the matrix $Y$ constructed there differs from $X$ in a single column in either $A$ or just one of the blocks $B_{i}$.

For example the UOMs

$$
\begin{aligned}
& {\left[\begin{array}{c}
a \\
a^{\prime}
\end{array}\right] \models\left(\left[\begin{array}{ccc}
b & c & d \\
b^{\prime} & e & f^{\prime} \\
g^{\prime} & c^{\prime} & f \\
g & e^{\prime} & d^{\prime}
\end{array}\right],\left[\begin{array}{ccc}
u & v & w \\
u^{\prime} & x & y^{\prime} \\
z^{\prime} & v^{\prime} & y \\
z & x^{\prime} & w^{\prime}
\end{array}\right]\right),} \\
& {\left[\begin{array}{ccc}
a & b & c \\
a^{\prime} & d & e^{\prime} \\
f^{\prime} & b^{\prime} & e \\
f & d^{\prime} & c^{\prime}
\end{array}\right] \models\left(\left[\begin{array}{c}
u \\
u^{\prime}
\end{array}\right],\left[\begin{array}{c}
v \\
v^{\prime}
\end{array}\right],\left[\begin{array}{c}
w \\
w^{\prime}
\end{array}\right],\left[\begin{array}{l}
x \\
x^{\prime}
\end{array}\right]\right)}
\end{aligned}
$$

are maximal in $\mathcal{O}(8,4)$. The first one is reducible and the second one irreducible.

We remark that all UOM-equivalence classes can be derived from the knowledge of the maximal classes. To explain how this is done we need to define some subsets of $\mathcal{O}(m, n)$ which we call "levels". The level $l$ of $\mathcal{O}(m, n)$ is the set $\{X \in \mathcal{O}(m, n): \nu(X)=l\}$. (The function $\nu$ is defined in Sec. II]) For convenience, we say that $X \in \mathcal{O}(m, n)$ lies on level $l$ if $\nu(X)=l$. Note that if $X$ lies on level $l$ then the whole equivalence class $[X]$ also lies on the same level.

Sketch of the algorithm. We first choose a set of representatives, say $R$, of the set of maximal classes. Next, from $R$ we extract the matrices which lie on the highest level, say $l$. To each $X$ in the selected set, we apply the following procedure. We construct all matrices $Y$ on level $l-1$ such that $Y \prec X$. There are only finitely many such $Y$ s. We discard those $Y$ which are not UOM, say by using Lemma 4, If no $Y$ s are left, then the class $[X]$ is minimal. After performing this procedure on all maximal $X$ on level $l$, we test the $Y$ s for equivalence. If two $Y$ s are equivalent, we remove one of them. We repeat this step until the remaining $Y$ s become pairwise non-equivalent. Note that none of these $Y \mathrm{~s}$ is maximal. Next, we enlarge the set of $Y \mathrm{~s}$ by adding the matrices in $R$ which lie on level $l-1$ (if any). Then we repeat the same procedure on the new set of $Y$ s that we constructed. We carry out this process to its end where no new matrices show up and all matrices of $R$ have been used up. The computation is admittedly tedious but it could be programmed to perform all steps on the computer. One of the hard steps is the test of equivalence.

\section{ACKNOWLEDGEMENTS}

LC was supported by Beijing Natural Science Foundation (4173076), the NNSF of China (Grant No. 11501024), and the Fundamental Research Funds for the Central Universities (Grant Nos. KG12001101, ZG216S1760 and ZG226S17J6). The second author was supported in part by the National Sciences and Engineering Research Council (NSERC) of Canada Discovery Grant 5285.

[1] N. Alon and L. Lovsz, Unextendible product bases. J. Combinatorial Theory, Ser. A, 95:169179, 2001.

[2] C. H. Bennett, D. P. DiVincenzo, T. Mor, P. W. Shor, J. A. Smolin, and B. M. Terhal, Unextendible product bases and bound entanglement, Phys. Rev. Lett.82, 5385 (1999).

[3] S. B. Bravyi, Unextendible product bases and locally unconvertible bound entangled states, Quantum Information Processing, 3, 309 (2004).

[4] Jianxin Chen and Nathaniel Johnston, The Minimum Size of Unextendible Product Bases in the Bipartite Case (and Some Multipartite Cases), Commun. Math. Phys. 333, 351-365 (2015). 
[5] Lin Chen and D.Ž. Đoković, Distillability and PPT entanglement of low rank quantum states, J. Phys. A: Math. Theor. 44, 285303 (2011), (26pp).

[6] L. Chen and D. Ž. Đoković, Qubit-qudit states with positive partial transpose, Phys. Rev. A 86, 062332 (2012).

[7] L. Chen and D. Ž. Đoković, Separability problem for multipartite states of rank at most 4, J. Phys. A. Math. Theor. 46 275304 (2013).

[8] L. Chen and D. Ž. Đoković, Orthogonal product bases of four qubits, J. Phys. A: Math. Theor. 50 (2017) 395301 (33pp)

[9] L. Chen and D. Ž. Đoković, Nonexistence of $n$-qubit unextendible product bases of size $2^{n}-5$, quant-ph/1709.01232 (2017).

[10] D. P. DiVincenzo, T. Mor, P. W. Shor, J. A. Smolin, and B. M. Terhal, Unextendible product bases, uncompletable product bases and bound entanglement, Commun. Math. Phys. 238, 379-410 (2003).

[11] Keqin Feng, Unextendible product bases and 1-factorization of complete graphs, Discrete Appl. Mathematics 154 (2006) 942-949.

[12] Y. Feng and Y. Shi, Characterizing locally indistinguishable orthogonal product states, IEEE Trans. Inform. Theory $\mathbf{5 5}$ (2009).

[13] Otfried Guhne, and Michael Seevinck, Separability criteria for genuine multiparticle entanglement, New J. Phys. 12, 053002 (2010).

[14] J. Harris, Algebraic Geometry, A First Course. Springer-Verlag, New York, 1992.

[15] Kyung Hoon Han and Seung-Hyeok Kye, Construction of multi-qubit optimal genuine entanglement witnesses, J. Phys. A 49, 175303 (2016).

[16] N. Johnston, The minimum size of qubit unextendible product bases. In Proceedings of the 8th Conference on the Theory of Quantum Computation, Communication and Cryptography (TQC) (2013).

[17] N. Johnston, The structure of qubit unextendible product bases, J. Phys. A. Math. Theor. 47, 424034 (2014).

[18] N. Johnston, 2014, Complete characterization of all unextendible product bases on 4 qubits www.njohnston.ca/4qubitupbs.txt

[19] B. Kraus, J. I. Cirac, S. Karnas, and M. Lewenstein, Phys. Rev. A 61, 062302 (2000).

[20] J. Tura, R. Augusiak, P. Hyllus, M. Kus, J. Samsonowicz, M. Lewenstein, Four-qubit entangled symmetric states with positive partial transpositions, Phys. Rev. A 85, 060302 (2012).

\section{APPENDIX A}


Table IV: UOMs of new sizes

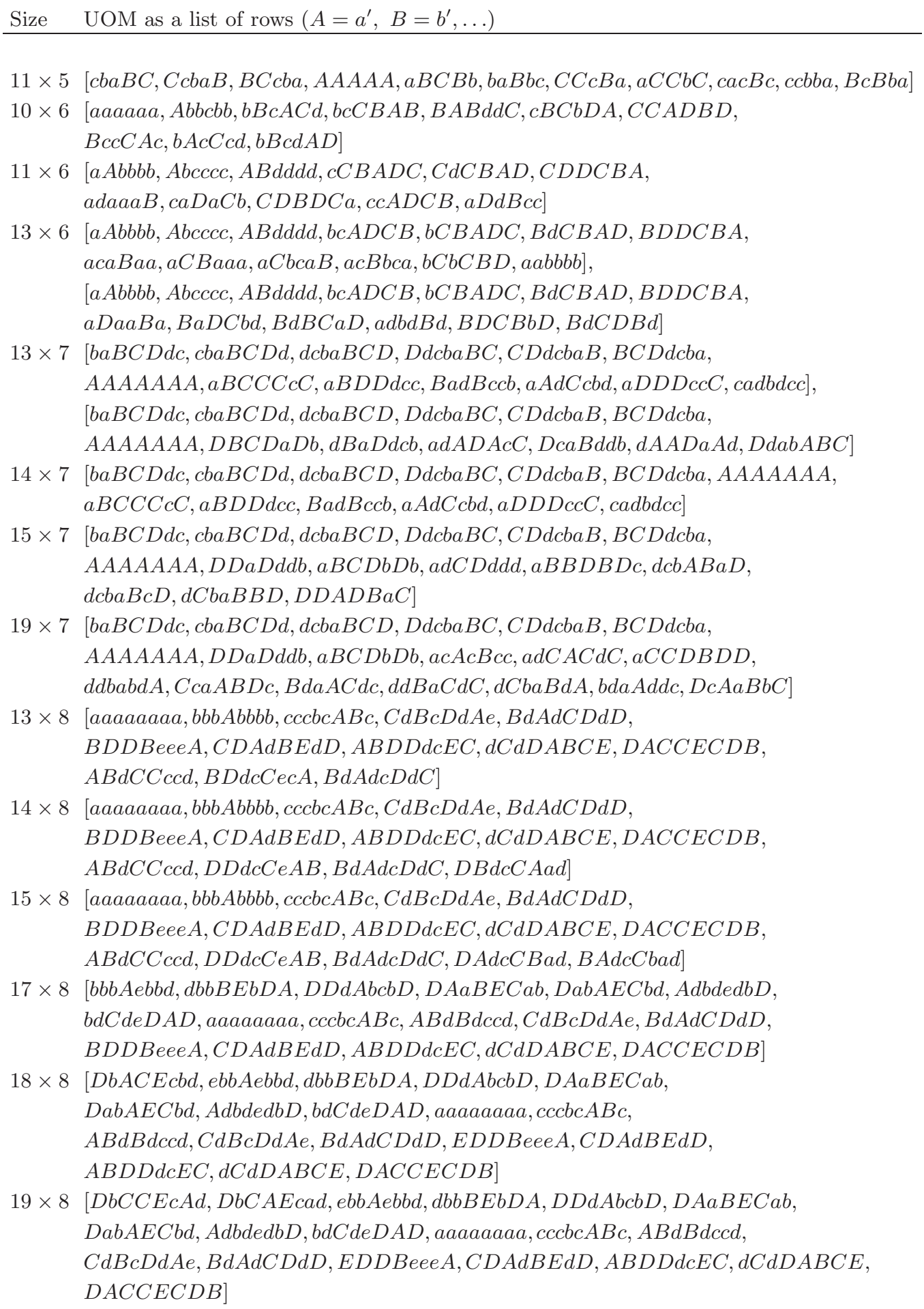

\section{APPENDIX B}

Corollary 16 shows that if $\mathcal{U}$ is a UPB in a multiqubit system and $\mathcal{U}^{\prime} \subset \mathcal{U},\left|\mathcal{U}^{\prime}\right|=|\mathcal{U}|-1$, then the subspace $\mathcal{U}^{\prime \perp}$ contains only finitely many product vectors. We show here that this fact is valid in arbitrary finite-dimensional quantum systems. Thus in this appendix we drop the condition that $\operatorname{Dim} \mathcal{H}_{i}=2$ for all $i$, and we set $d_{i}=\operatorname{Dim} \mathcal{H}_{i}$ for $i=1,2, \ldots, n$. Then $d:=\prod d_{i}$ is the dimension of $\mathcal{H}$. 
Moreover, the fact mentioned above remains valid when we replace UPBs with generalized UPBs. They are defined as follows.

A generalized $U P B$ (abbreviated as gUPB) is a linearly independent set $\mathcal{U} \subset \mathcal{H}$ of unit product vectors such that $\mathcal{U}^{\perp}$ contains no product vector.

Proposition 19 Let $\mathcal{U}$ be a $g U P B$ of cardinality $m$ in $\mathcal{H}$ and let $\mathcal{U}^{\prime}$ be a subset of $\mathcal{U}$ of cardinality $m-1$. Then the subspace $\mathcal{U}^{\perp}$ contains only finitely many product vectors (up to scalar multiples).

Proof. The Segre variety $\mathcal{S}:=P\left(\mathcal{H}_{1}\right) \times \cdots \times P\left(\mathcal{H}_{n}\right)$ is embedded in the complex projective space $P(\mathcal{H})$ as a closed subvariety. The projective linear subspaces $\Lambda:=P\left(\mathcal{U}^{\perp}\right)$ and $P\left(\mathcal{U}^{\prime \perp}\right)$ of $P(\mathcal{H})$ have dimensions $d-m-1$ and $d-m$, respectively. Set $X:=P\left(\mathcal{U}^{\perp}\right) \cap \mathcal{S}$, a closed subvariety of $P\left(\mathcal{U}^{\prime \perp}\right)$, and let $k$ be its dimension.

Assume that $k \geq 1$. By applying [14, Proposition 11.4] to the projective space $P\left(\mathcal{U}^{\prime \perp}\right)$ of dimension $d-m$, its subvariety $X$ of dimension $k$, and the linear subspace $\Lambda$ of dimension $d-m-1$, we deduce that $\Lambda$ must intersect $X$. As $X \subseteq \mathcal{S}, \Lambda$ also intersects $\mathcal{S}$. This contradicts the hypothesis that $\mathcal{U}$ is a gUPB. We conclude that $k<1$, i.e., $X$ must be a finite set (possibly empty).

As an example let us consider the case of two qutrits: $n=2, d_{1}=d_{2}=3, d=9$. Let $\mathcal{U}$ be the Pyramid UPB in $\mathcal{H}$ (see e.g., [10]). It consists of five product states $\psi_{i}=v_{i} \otimes v_{2 i}(\bmod 5), i=0, \ldots, 4$, where

$$
v_{i}=N\left(\cos \frac{2 \pi i}{5}, \sin \frac{2 \pi i}{5}, h\right), \quad i=0, \ldots, 4
$$

with $h=\frac{1}{2} \sqrt{1+\sqrt{5}}$ and $N=2 / \sqrt{5+\sqrt{5}}$. Let $\mathcal{U}^{\prime}$ be the OPS consisting of the four states $\psi_{i}, i=0, \ldots, 3$. Denote by $\rho$ the projector associated with $\mathcal{U}^{\prime}$. One can verify that the range of $\rho$ contains exactly six product states. To write down these product states, we denote by $u_{i, j}, i \neq j$, the unit vector orthogonal to $v_{i}$ and $v_{j}$. Then the six product vectors in the range of $\rho$ are $\psi_{4}=v_{4} \otimes v_{3}, u_{2,4} \otimes v_{3}$ and the four mutually orthogonal states $v_{0} \otimes u_{0,2}, v_{3} \otimes u_{1,4}$, $u_{1,3} \otimes v_{2}, u_{0,2} \otimes v_{4}$. None of the last four states is orthogonal to $\psi_{4}$ or $u_{2,4} \otimes v_{3}$. By using a computer we have verified that $\rho$ and the density matrices of the six unit product vectors in the range of $\rho$ are linearly independent. It follows that $\rho$ is not separable. To summarize, $\rho$ is a (non-normalized) PPTES of rank 5 whose range contains exactly 6 product vectors.

While in the multiqubit case we can easily find all product vectors in the range of $\rho$, this example shows that in general this task is not easy. 